\title{
Measurement of Optical Coefficients of Tissue-like Solutions using a Combination Method of Infinite and Semi-infinite Geometries with Continuous Near Infrared Light
}

\author{
Wooseok Ko, Yoonkeun KwAK and Soohyun Kiм* \\ Department of Mechanical Engineering, Korea Advanced Institute of Science and Technology, \\ Guseong-dong, Yuseong-gu, Daejeon 305-701, Korea
}

(Received April 13, 2006; revised June 20, 2006; accepted June 22, 2006; published online September 7, 2006)

\begin{abstract}
This paper proposes a new continuous wave $(\mathrm{CW})$ method to measure the optical coefficients of a tissue-like solution by using a combination of infinite and semi-infinite geometries. Previous CW methods have required a long source-detector separation distance to distinguish the effects of absorption from scattering. This long separation distance results in inaccurate optical coefficients due to a low signal-to-noise ratio (SNR). A combination method is suitable for measuring optical coefficients of tissue-like solutions while using a short source-detector distance to increase the accuracy of the CW method. The measurement geometries are switched by an optical system, and light is detected at an avalanche photo-detector (APD). The absorption and reduced scattering coefficients are calculated from the slope and intercept values of a straight line derived from the fluence rate at several source-detector distances. The combination method provides an excellent separation performance between scattering and absorption with continuous near infrared (NIR) light. [DOI: 10.1143/JJAP.45.7158]
\end{abstract}

KEYWORDS: NIR light, absorption, scattering, phantom, intralipid, continuous wave

\section{Introduction}

Over the last decade, considerable progress has been made toward developing a novel biomedical imaging technique using near-infrared (NIR) light to probe biomedical tissue. In the NIR optical window, from 700 to $900 \mathrm{~nm}$, light can penetrate tissue up to several centimeters, because protein and water have a very small absorption coefficient for NIR light. The principal NIR absorbers are assumed to be deoxygenated hemoglobin $(\mathrm{Hb})$ and oxygenated hemoglobin $\left(\mathrm{Hb}-\mathrm{O}_{2}\right) .{ }^{1-3)}$ NIR spectroscopy offers a safe way to quantify these components and to view unique functional information with low-cost "point-of-care" devices. Successful application of NIR in the medical field requires understanding and knowledge about various tissue-like solutions. Therefore, the method development for measuring the optical coefficients of tissue-like solutions is important for tissue diagnostics, patient monitoring, and image reconstruction with NIR light.

Multiple light scattering inside the tissue-like solution complicates quantitative measurements of light absorption in the NIR region. ${ }^{2,3)}$ The NIR device needs a technique to separate the effects of absorption from those of scattering. Fundamentally, the absorption coefficient $\mu_{\mathrm{a}}$ and reduced scattering coefficient $\mu_{\mathrm{s}}^{\prime}$ can be determined by using frequency-domain (FD), time-domain (TD), or continuous wave $(\mathrm{CW})$ measurement with a diffusion model. The FD method, which requires a light intensity modulated at a certain radio frequency, measures the phase delay of a modulated light source between source and detector, the average intensity attenuation, and the intensity oscillation amplitude. Using these measurements at several sourcedetector distances, the optical coefficients can be calculated. ${ }^{3-7)}$ The TD method measures the time-of-flight distribution of a light pulse, of which the shape is dependent on optical propeties. ${ }^{8,9)}$ Both FD and TD methods require a tunable laser or an extensive assembly of laser diodes and a highly sensitive detector. Both methods also have the drawbacks of being expensive and sophisticated to construct system. For FD and TD methods, CW technique is

*E-mail address: soohyun@kaist.ac.kr comparatively less expensive, easy to use, and suitable for the determination of $\mu_{\mathrm{a}}$ and $\mu_{\mathrm{s}}^{\prime}$ over a wide range of wavelength. ${ }^{1,10)}$ In recent years, developing a measurement technique for the optical coefficients of tissue-like solutions using CW light has become an important goal. Traditional CW methods have used a large separation distance to find optical coefficients. ${ }^{1)}$ However, performance in measuring optical coefficients is limited due to low signal-to-noise ratio (SNR) induced by a large separation distance. In this paper, a combination method using infinite and semi-infinite geometries is proposed to provide an excellent separation performance for optical coefficients with $\mathrm{CW}$ light.

\section{Diffusion Equation}

\subsection{Background}

When working in a turbid medium, such as a tissue-like solution, it is useful to regard light propagation as a photon diffusion process. Wave intensity is attenuated while penetrating tissue, but polarization is neglected. The fluence rate at a specific position can be accurately calculated from the diffusion approximation to the Boltzmann transport equation when the measurement position is far from the sources or boundaries and absorption in the medium is low. The steady stated diffusion equation for a point source can be expressed as follows. ${ }^{11}$ )

$$
-D \nabla^{2} \phi(\boldsymbol{r})+\mu_{\mathrm{a}} \phi(\boldsymbol{r})=S(\boldsymbol{r})
$$

Here, $\phi(\boldsymbol{r})$ is the fluence rate $\left(\mathrm{W} / \mathrm{m}^{2}\right), D=1 / 3\left(\mu_{\mathrm{a}}+\mu_{\mathrm{s}}^{\prime}\right)$ is the diffusion constant, and $S\left(\mathrm{~W} / \mathrm{m}^{2}\right)$ is the light source term. In the strongly scattering media, the diffusion constant $D$ can be defined as $1 / 3 \mu_{\mathrm{s}}^{\prime}$. The solution for the infinite medium, as shown in Fig. 1(a), is easily built from Green's function, because the light source can be assumed as an isotropic point source in an infinite, homogeneous medium. Then, a detected fluence of continuous light at a separation $r$ from the source is given as

$$
\phi(r)=\frac{A}{4 \pi D} \frac{\exp \left(-\mu_{\mathrm{eff}} r\right)}{r}
$$

where $A$ is the strength of the light source, and $\mu_{\text {eff }}$ is represented as $\sqrt{3 \mu_{\mathrm{a}}\left(\mu_{\mathrm{a}}+\mu_{\mathrm{s}}^{\prime}\right)}$. 


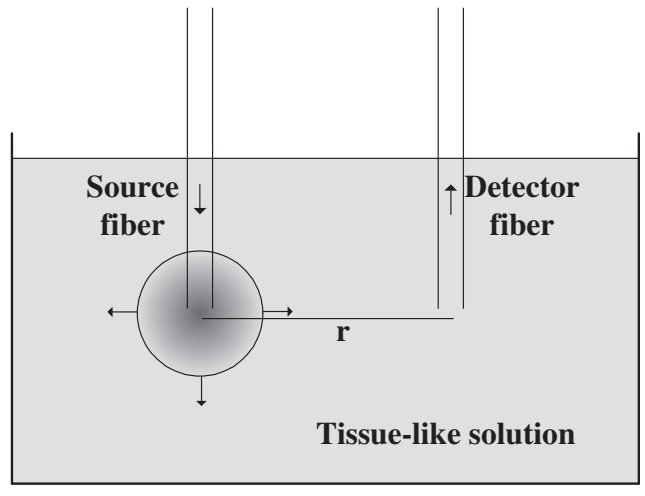

(a)

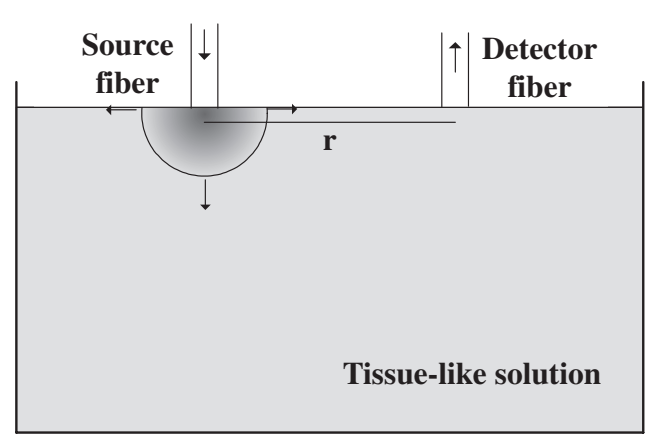

(b)

Fig. 1. The measurement configuration for (a) infinite and (b) semi-infinite geometry. The detector fiber is oriented perpendicular to the radial flux from the source for (a). Therefore, the detected signal is simply proportion to the fluence rate.

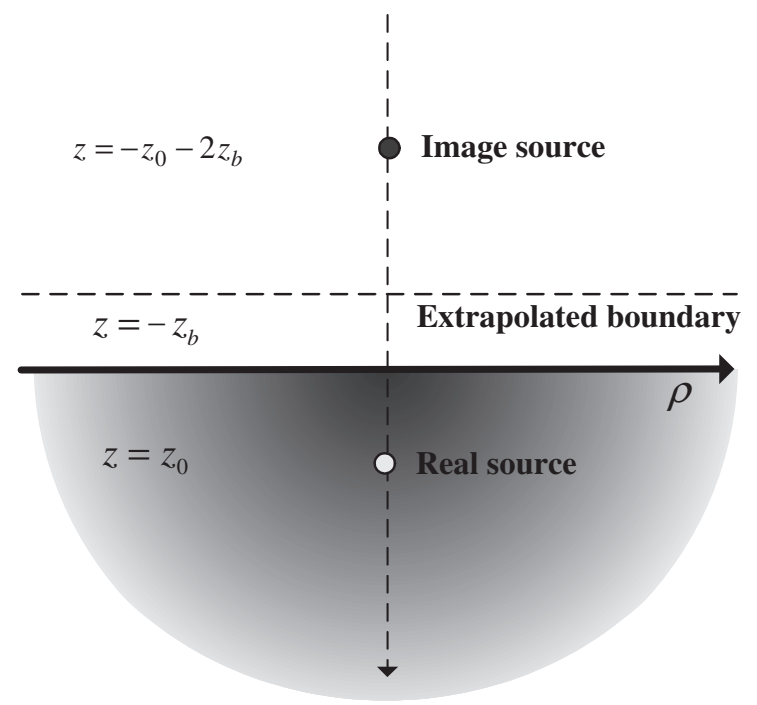

Fig. 2. The position of the real source and the image source for a extrapolated boundary condition.

To obtain a solution for the semi-infinite geometry as shown in Fig. 1(b), a different boundary condition is required. For a photon source at a depth $z_{0} \approx 1 / \mu_{\mathrm{s}}^{\prime}$, there will be a negative image source at $-z_{0}-2 z_{\mathrm{b}}$, forcing the fluence to zero on the extrapolated boundary at $-z_{\mathrm{b}}$, as shown in Fig. 2. The extrapolated boundary quantity is represented as follows. ${ }^{12)}$

$$
z_{\mathrm{b}}=\frac{1+R_{\mathrm{eff}}}{1-R_{\mathrm{eff}}} \cdot \frac{2}{3 \mu_{\mathrm{s}}^{\prime}}
$$

Here, $R_{\text {eff }}$ is the fraction of photons that is internally diffusely reflected at the boundary. Haskell et al. found $R_{\text {eff }}$ values according to the various refractive indices of a semiinfinite medium. ${ }^{12)}$ The solution at a point $(\rho, z)$ due to a source at position $\left(\rho, z_{0}\right)$ in the semi-infinite medium is given by summing Green's function for the real source and the image source, as follows. ${ }^{13)}$

$$
\phi(\rho, z)=\frac{A}{4 \pi D}\left(\frac{\exp \left(-\mu_{\mathrm{eff}} r_{1}\right)}{r_{1}}-\frac{\exp \left(-\mu_{\mathrm{eff}} r_{2}\right)}{r_{2}}\right)
$$

In eq. (4),

$$
\begin{aligned}
& r_{1}=\sqrt{\left(z-z_{0}\right)^{2}+\rho^{2}} \\
& r_{2}=\sqrt{\left(z+z_{0}+2 z_{\mathrm{b}}\right)^{2}+\rho^{2}}
\end{aligned}
$$

For the semi-infinite measurement, a photon current leaving the tissue at $z=0$ is considered as ${ }^{14)}$

$$
R(\rho)=-\left.D \hat{n} \cdot \nabla \phi(\rho, z)\right|_{z=0}=-\left.D \frac{\partial \phi}{\partial z}\right|_{z=0}
$$

where $R$ is the reflectance emitted from a semi-infinite surface at a separation $\rho$ from the source, $\hat{n}$ is a vertical unit vector to the surface. From eqs. (4)-(6), the diffuse reflectance can be obtained as follows.

$$
R(\rho, z=0)=\frac{A}{4 \pi}\left[\frac{1}{\mu_{\mathrm{s}}^{\prime}}\left(\mu_{\mathrm{eff}}+\frac{1}{r_{10}}\right) \frac{\exp \left(-\mu_{\mathrm{eff}} r_{10}\right)}{r_{10}^{2}}+\left(\frac{1}{\mu_{\mathrm{s}}^{\prime}}+\frac{2}{\mu_{\mathrm{s}}^{\prime}} z_{\mathrm{b}}^{\prime}\right)\left(\mu_{\mathrm{eff}}+\frac{1}{r_{20}}\right) \frac{\exp \left(-\mu_{\mathrm{eff}} r_{20}\right)}{r_{20}^{2}}\right]
$$

where $r_{10}$ and $r_{20}$ are the values of $r_{1}$ and $r_{2}$ with $z=0$ in eq. (4), and $z_{\mathrm{b}}^{\prime}$ is given as follows.

$$
z_{\mathrm{b}}^{\prime}=\frac{2}{3} \frac{1+R_{\mathrm{eff}}}{1-R_{\mathrm{eff}}}
$$

\subsection{Measurement model}

The CW method generally requires multiple sourcedetector separation distances. By measuring the photon fluence emitting from a tissue-like solution at short and long separation distances, the two optical coefficients can be calculated. However, the large separation distance distorts the detected signal and degrades CW measurement accuracy. The proposed combination method can distinguish the two optical coefficients with linear relationships between the fluence rate and the separation distance in infinite and semiinfinite geometries. Equation (2) yields following linear equation. 


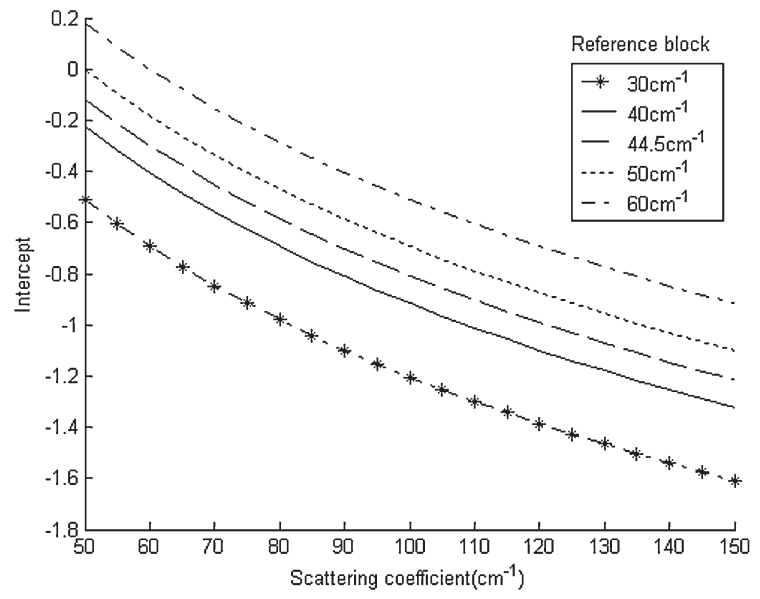

(a)

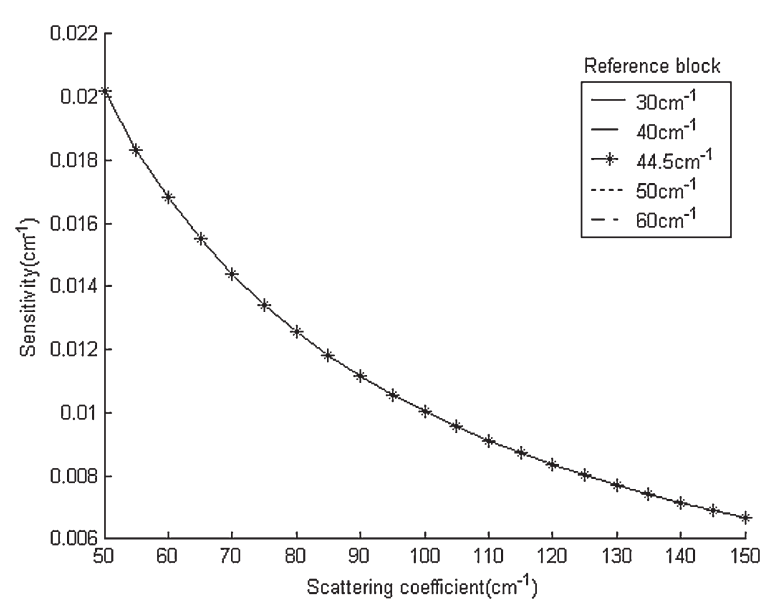

(b)

Fig. 3. (a) Intercept value and (b) measurement sensitivity of the proposed method according to the reference silicone block having the various scattering coefficients. The measurement sensitivity is the ratio of the change in intercept to the change of the scattering coefficient.

$$
\ln (r \phi(\rho, z))=-\mu_{\mathrm{eff}} \cdot r+\ln \left(\frac{A}{4 \pi D}\right)
$$

Equation (9) has been experimentally proven, and it provides a good description of the homogeneous infinite medium problem in a multiple scattering regime. ${ }^{11-14)} \mu_{\text {eff }}$ value is a slope value of a straight line derived from the fluence rate at several source-detector distances in eq. (9).

Equation (7) can also be approximated as follows using an assumption, $r_{10} \approx r_{20} \approx \rho$, because $\rho$ is as much $20-30$ times large as $z_{0}$ at $1 \mathrm{~cm}$ separation.

$$
R(\rho, z=0)=\frac{A^{\prime}}{\mu_{\mathrm{s}}^{\prime}}\left(\mu_{\mathrm{eff}}+\frac{1}{\rho}\right) \frac{\exp \left(-\mu_{\mathrm{eff}} \rho\right)}{\rho^{2}}
$$

Here, $A^{\prime}=A /\left(2 \pi\left(1+z_{\mathrm{b}}^{\prime}\right)\right)$ has a constant value. $A^{\prime}$ needs to be measured every experiment to determine a scattering coefficient with a semi-infinite boundary condition. To solve this problem, the proposed combination method uses a reference silicone block with known optical coefficients. Another linear model is derived in terms of the reflectance ratio between the tissue-like solution and the reference silicone block, as follows.

$$
\begin{aligned}
\ln \left(\frac{R}{R_{\text {ref }}}\right)= & \left(\mu_{\text {e_ref }}-\mu_{\text {eff }}\right) \rho+\ln \left(\frac{A^{\prime}}{A_{\text {ref }}^{\prime}} \frac{\mu_{\text {s_ref }}^{\prime}}{\mu_{\text {s }}^{\prime}}\right) \\
& +\ln \left[\frac{\mu_{\text {eff }}+(1 / \rho)}{\mu_{\text {e_ref }}+(1 / \rho)}\right]
\end{aligned}
$$

Here, $R_{\text {ref }}, \mu_{\text {s_ref }}^{\prime}, \mu_{\text {e_ref }}$, and $A_{\text {ref }}^{\prime}$ are the coefficients for the reference silicone block. Equation (11) has $\ln A^{\prime} \mu_{\mathrm{s} \_ \text {ref }}^{\prime} / A_{\text {ref }}^{\prime} \mu_{\mathrm{s}}^{\prime}$ as an intercept, making $\ln \left(\left(\mu_{\text {eff }}+1 / \rho\right) /\left(\mu_{\text {e_ref }}+1 / \rho\right)\right)$ zero when $\rho \approx 0$. The intercept value can be defined as $\ln \mu_{\text {s_ref }}^{\prime} /$ $\mu_{\mathrm{s}}^{\prime}$ using a reference block having similar refractive index with the tissue-like solutions, because $A^{\prime}$ value depends on the refractive index and the strength of the light source. Absorption and reduced scattering coefficient of a tissue-like solution is computed from the slope and intercept from eqs. (9) and (11). In this paper, a silicone rubber block with optical coefficients $\mu_{\mathrm{a}}=0.028 \mathrm{~cm}^{-1}$ and $\mu_{\mathrm{s}}^{\prime}=44.5 \mathrm{~cm}^{-1}$ at $632.8 \mathrm{~nm}$ was used. The silicone block has the $R_{\text {eff }} 0.4980$

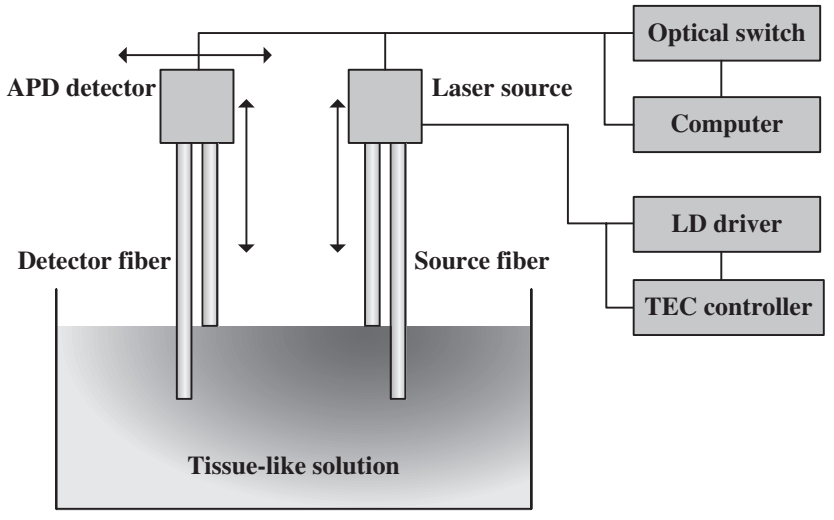

Fig. 4. Experimental arrangement with continuous NIR light for the combination method.

with the refractive index 1.36. Figures 3(a) and 3(b) show the change of intercept value and the measurement sensitivity, which is the ratio of the change in intercept to the change of the scattering coefficients, according to the reference block having various optical properties. Figure 3 (b) shows the optical coefficients of the reference silicone block are not important in measuring the change of the scattering coefficient because influence of sensitivity difference is extremely small as in eq. (9).

\section{Experimental Results}

The combination model was experimentally tested using a macroscopically homogeneous medium with a strong scattering coefficient. Figure 4 depicts the experimental setup. An laser diode (LD) driver (Thorlabs IP500) and a temperature controller (Thorlabs CM100T) are used to maintain a selected wavelength and constant power of the light source (635 nm LD, Hitachi $10 \mathrm{~mW}$ HL6320G). Light is transmitted through an optical fiber (3M FT-400UMT) to the tissue-like solution. An avalanche photo diode (APD; Hamamatsu C5460-01) detects the intensity of NIR light emitting from the solution with high sensitivity. The detector fiber is oriented perpendicular to the radial flux from the source. 


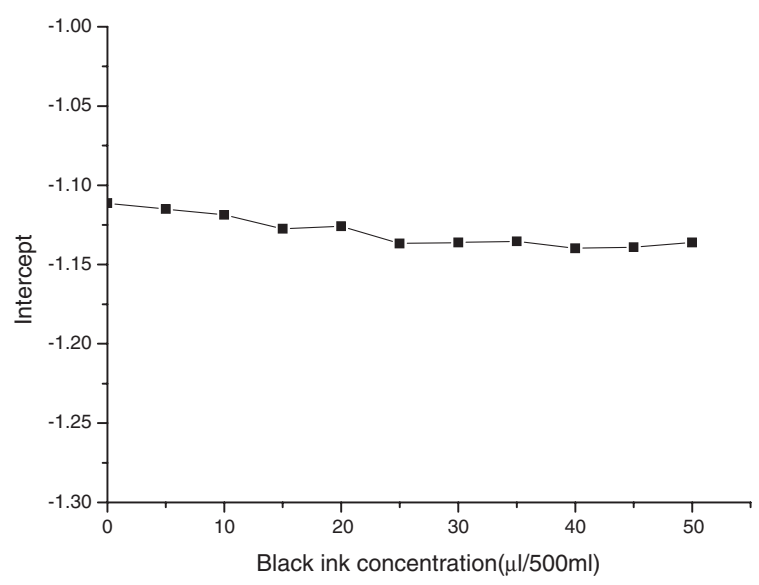

(a)

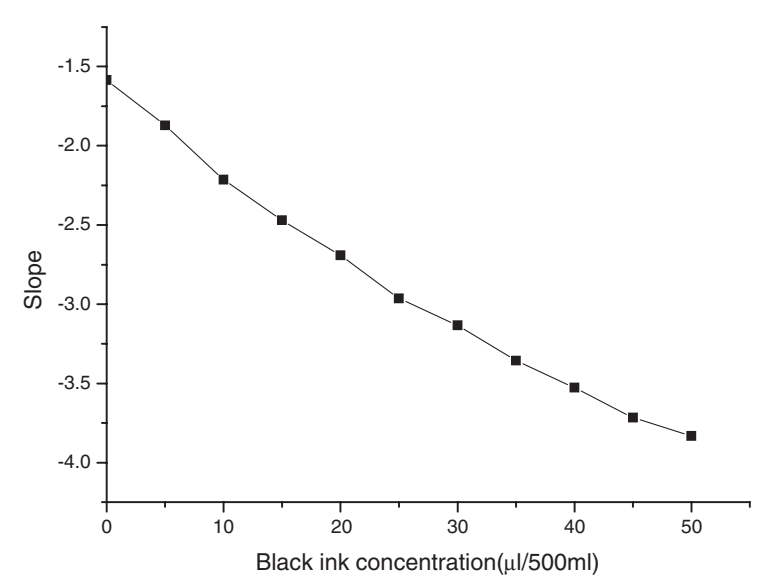

(b)

Fig. 5. (a) Slopes and (b) intercepts calculated from light intensity measured in two boundary conditions as a function of black ink concentration.

Therefore, the detected signal is simply proportion to the fluence rate in eq. (7) for the infinite geometry configuration. The distance between the source and the detector is varied from 0.5 to $3 \mathrm{~cm}$. In the combination model, an optical switch selects an infinite or semi-infinite geometry configuration and light emitting from a scattering medium is analyzed according to different source-detector distances. The absorption and scattering coefficients are calculated from the slope and intercept of the straight line computed with eqs. (7) and (9). The total acquisition time for measuring the optical coefficients is below $10 \mathrm{~s}$.

In this experiment, an intralipid-10\% solution (Fresenius Kabi) and black Indian ink are used. The intralipid has been assumed as an ideal liquid phantom of human in previous research. $^{3,5)}$ The intralipid solution is mixed with the diluted Indian ink solution to obtain various absorption coefficients. Figures 5(a) and 5(b) indicate that increasing ink amount in the scattering solution led to slope change in positive side, while the intercept remained almost unchanged, less than $1 \%$. Thus, it is possible to separate the effects of scattering and absorption with the slop and intercept values by using the suggested combination method. The measured absorption and scattering coefficients without ink are 0.0062 and $135.2 \mathrm{~cm}^{-1}$ respectively. The obtained optical coefficients are compared with those of three different methods, as

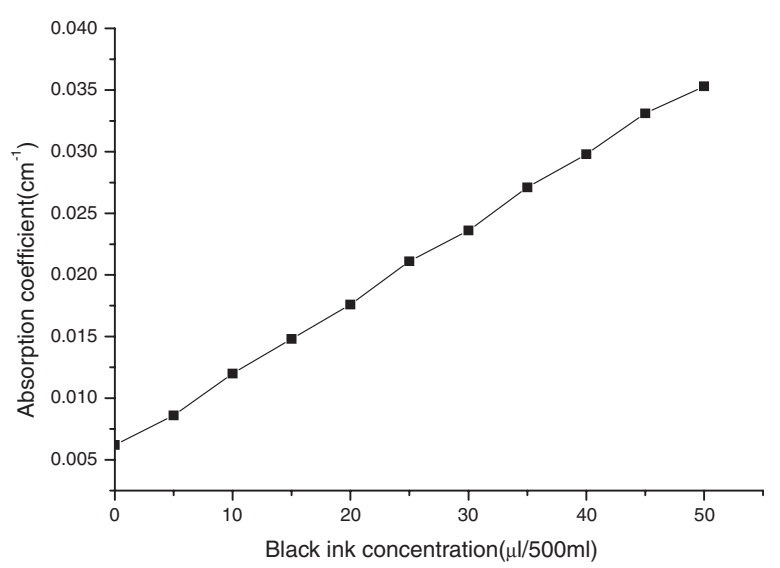

(a)

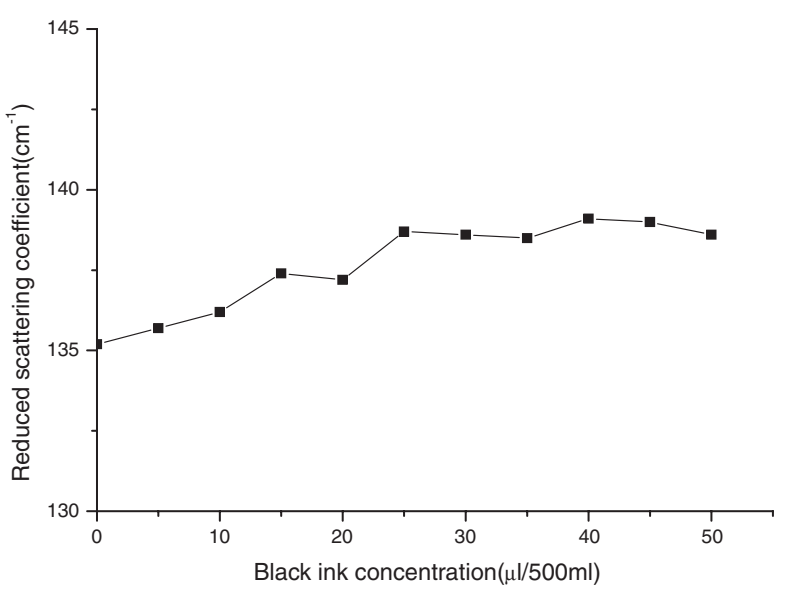

(b)

Fig. 6. (a) Absorption and (b) scattering coefficient measured for different black ink concentration.

Table I. Experimental values of absorption and reduced scattering coefficients for intralipid-10\% compared with the experimental values of other investigations.

\begin{tabular}{llc}
\hline & $\begin{array}{c}\mu_{\mathrm{a}} \\
\left(\mathrm{cm}^{-1}\right)\end{array}$ & $\begin{array}{c}\mu_{\mathrm{s}}^{\prime} \\
\left(\mathrm{cm}^{-1}\right)\end{array}$ \\
\hline Combination method & 0.006 & 135.2 \\
Moes et al. ${ }^{15)}$ & 0.6 & 105.85 \\
Flock et al. ${ }^{16)}$ & 0.0018 & 59.5 \\
van Staveren et al. ${ }^{17)}$ & 0.149 & 115.5
\end{tabular}

shown in Table I. ${ }^{15-17)}$ The gap between our experimental results and those of previous studies are partly attributed to a difference in intrtalipid-10\% composition. Figures 6(a) and 6(b) show the absorption and reduced scattering coefficient according to various concentrations of black ink. The absorption coefficients are in proportion to black ink concentration, as shown in Fig. 6(a). In principle, the addition of ink into intralipid solution changes mainly the absorption coefficient of the sample solution without influencing its scattering coefficient. Figure 6(b) indicates that the systematic uncertainties involved in using the $\mathrm{CW}$ method influence on the change of the reduced scattering coefficients with \pm 1.4 around $136.5 \mathrm{~cm}^{-1}$. 


\section{Conclusions}

Several instruments based on the $\mathrm{CW}$ method have been proposed to measure the absorption and scattering coefficients of tissue-like solutions. Although some of the proposed instruments have been fairly successful, most of them have a major problem, namely, that the long sourcedetector distance distorts the output signal. In this paper, a new method, which combines semi-infinite and infinite geometries to separate the absorption effect from scattering, is proposed. This method calculates optical coefficients from the slope and intercept of the linear diffusion model. Experimental results show that the proposed method is remarkable in distinguishing the optical coefficients. This is a strong indication that the proposed experimental method is suitable for measurement with the tissue-like sample by using CW NIR light.

\section{Acknowledgement}

This work was carried out at Korea Advanced Institute of Science and Technology (KAIST), which was supported by the Korea Research Foundation Grant funded by the Korean Government (MOEHRD, Basic Research Promotion Fund) (KRF-2005-015-C00173).
1) P. Rolfe: Annu. Rev. Biomed. Eng. 2 (2000) 715.

2) A. Ishimaru: Appl. Opt. 28 (1989) 2210

3) E. Gratton, S. Fantini, M. A. Franceschini, G. Gratton and M. Fabiani: Philos. Trans. R. Soc. London, Ser. B 352 (1997) 727.

4) B. W. Pogue and M. S. Patterson: Phys. Med. Biol. 39 (1994) 1157.

5) S. Fantini, M. A. Franceschini, J. S. Maier, S. A. Walker, B. Barbieri and E. Gratton: Opt. Eng. 34 (1995) 32.

6) T. H. Phan, O. Coquoz, J. B. Fishkin, E. Anderson and B. J. Tromberg: Rev. Sci. Instrum. 71 (2000) 2500

7) B. J. Tromberg, O. Coquoz, J. B. Fishkin, T. Pham, E. Anderson, J. Bulter, M. Cahn, J. D. Gross, V. Venugopalan and D. Pham: Philos. Trans. R. Soc. London 352 (1997) 967.

8) T. Kitai, M. Miwa, H. Liu, B. Beauvoit, B. Chance and Y. Yamaokak: Phys. Med. Biol. 44 (1999) 2049.

9) M. S. Patterson, B. Chance and B. C. Wilson: Appl. Opt. 28 (1989) 2331.

10) T. J. Farrel, M. S. Patterson and B. Willson: Med. Phys. 19 (1992) 879

11) S. Fantini, M. A. Franceschini and E. Gratton: J. Opt. Soc. Am. 11 (1994) 2128.

12) R. C. Haskell, L. O. Svaasand, T. T. Tsay, T. C. Feng, M. S. Mcadams and B. J. Tromberg: J. Opt. Soc. Am. A 11 (1994) 2727.

13) C. Cheung, J. P. Culver, K. Takahashi, T. H. Greenberg and A. G. Yodh: Phys. Med. Biol. 46 (2001) 2053.

14) A. Kienle and M. S. Patterson: J. Opt. Soc. Am. 14 (1997) 246.

15) C. J. M. Moes, M. J. C. van Gemert, W. M. Star, J. P. A. Marijnssen and S. A. Prahl: Appl. Opt. 28 (1989) 2292.

16) S. T. Flock, S. L. Jacques, B. C. Wilson, W. M. Star and M. J. C. van Gemert: Lasers Surg. Med. 12 (1992) 510.

17) H. J. van Staveren, C. J. M. Moes, J. van Marle, S. A. Prahl and M. J. C. van Gemert: Appl. Opt. 30 (1991) 4507. 